\title{
The ground impulse generated by a plane fuel-air explosion with side relief $\dagger$
}

\author{
M. SICHEL \\ Professor of Aerospace Engineering, The University of Michigan, Ann Arbor, MI 48109, U.S.A.
}

\author{
AND \\ J. C. FOSTER \\ Project Scientist, Eglin Air Force Base, FL 32542, U.S.A.
}

(Received 27 September 1977; Revised 26 May 1978)

\begin{abstract}
Detonations can be initiated in unconfined fuel-oxidizer clouds by blast waves of sufficient energy. The ground impulse generated by such detonations can cause considerable damage. An analytical theory is developed in the present paper, which makes it possible to predict this impulse, and experimental measurements which verify the theory are reported.

A plane detonation wave propagating through a one-dimensional fuel-air cloud in contact with the ground and with the inert atmosphere at height $h$ is considered. The wave is followed by an expansion wave which propagates from the inert gases into the combustion products, and an oblique shock is induced in the inert bounding the explosive. In computing the impulse only the region behind the detonation where the expansion is reflected from the ground is considered. The impulse per unit area is found to be the product of $p_{2}(h / C)$ and a universal impulse function $U_{1}(\xi)$, where $p_{2}$ is the riessure behind the detonation and $C$ is the Chapman-Jouguet velocity, and $\xi=x / l$ is the dimensionless distance from the detonation.

Experimental values of the pressure and impulse behind a plane wave were obtained using a plastic bag, $4 \times 4 \times 20 \mathrm{ft}$ long, filled with a stoichiometric MAPP-air mixture. The detonation was initiated using an explosive initiator at one end of the bag. Pressure transducers placed on the ground plane along the center of the bags were used to determine the variation of the pressure and impulse per unit area with time. Theory and experiment were found to be in excellent agreement.
\end{abstract}

\section{Introduction}

DETONATIONS can be initiated in unconfined fuel-air clouds by the rapid release of energy. The pressure and velocity induced behind such detonations can result in extensive damage. The present paper deals with the ground impulse generated in such fuel-air explosions.

In general the ground impulse will depend on the cloud shape, the fuel distribution within the cloud, and the location relative to the initiator. Idealized analytical models of such fuel-air explosions have been discussed by Sichel and $\mathrm{Hu}$ (1973). The calculation of the impulse generated by detonation of a centrally initiated hemispherical fuel-air cloud is a one dimensional unsteady problem and has been treated numerically by Fishburn (1976). Fishburn (1976) considers wave propagation both within the cloud and into the inert surrounding medium.

†Paper presented at Sixth International Colloquium on Gasdynamics of Explosions and Reactive Systems, 22-26 August 1977 
In practice the shape of fuel-air clouds, such as might result from fuel spills, is more likely to approximate a cylindrical disk rather than a hemisphere. In order to evaluate the characteristics of different fuel-air combinations, it is also frequently convenient to experiment with plane or one dimensional configurations by using rectangular plastic bags filled with the combustible mixture of interest. In these cases the presence of the inert boundary between the explosive fuel-air mixture and the surrounding inert air greatly modifies the pressure signature and ground impulse compared to that computed using one dimensional theory. Numerical computation of the two dimensional unsteady flows which result is complex and expensive; hence, simple approximate analyses are of great interest. A simple method of computing the ground impulse of a detonation with side relief is developed below, and the results are compared with experimental measurements.

In the first section below the nature of the problems involved in computing static impulse, even in the simple case of a fuel-air explosion with plane geometry are considered. A simple theory for computing ground impulse is then described followed by a comparison of the theory and corresponding experimental measurements.

\section{One dimensional fuel-air explosions}

The difficulties in analyzing fuel-air explosions (FAE) in clouds other than those of spherical symmetry can be demonstrated by considering the "one dimensional" clouds which arise in shock tube or bag tests. Such a cloud with direct initiation by a plane explosive charge at the center is shown in Fig. 1.

When the surfaces bounding the cloud are rigid, the FAE behaves like an infinite, plane explosion. The initiating charge first generates a strong, plane blast wave. For strong enough charges, transition to a plane detonation occurs when the blast radius $r_{s}$ is of the order of a critical radius $r^{*}$, defined by equating the blast energy to the heat of combustion released inside $r^{*}$. During this transition, which has been studied in part by Bach et al. (1971) and by Lee (1972), the blast wave changes to a detonation followed by a Taylor Expansion (Taylor, 1958). This self similar flow, for which analytical solutions have been found (Zeldovitch, 1942, Sedov, 1959), is also obtained numerically by Fishburn (1976) for the flow within the explosive cloud.

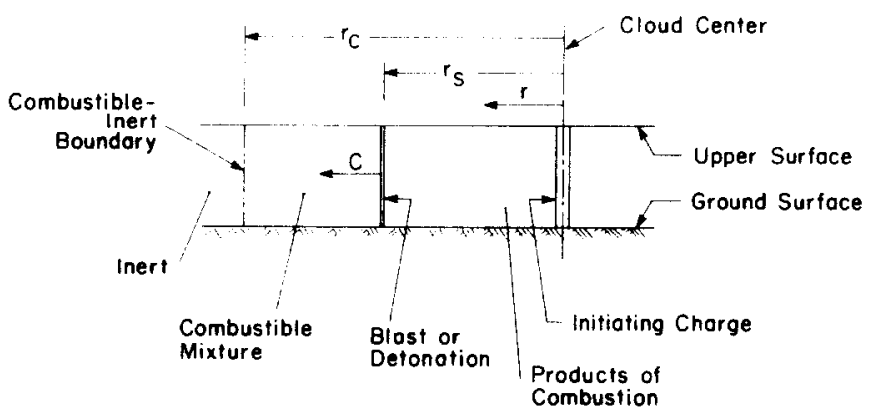

Fig. 1. One dimensional fuel-air explosions. 
When the detonation reaches the edge of the cloud $r=r_{c}$, the flow changes once again. A shock propagates into the inert gas beyond the fuel cloud, and a reflected expansion generally moves into the combustion products. While Fishburn (1976) does not consider the details of initiation, the other processes described here are taken into account by his calculations for a spherical cloud.

More typically the upper surface, and the sides in a cloud with a rectangular cross section, rather than being rigid, are bounded by an inert gas like air. Then it becomes necessary to deal with a two or even three dimensional unsteady flow. The initiating blast will no longer be plane because of the compressible boundary. Once a detonation is established, it will be followed by an expansion wave propagating from the inert boundary into the combustion products while an oblique shock is induced in the inert boundary gas as shown in Fig. 2 for a two dimensional cloud. The blast-detonation transition, and the propagation beyond the fuel cloud radius will, in this case, be quite complex and have not, to the authors' knowledge, been treated in detail.

The presence of the compressible boundary may also result in a decrease in the propagation velocity of the detonation. This effect, which has been investigated by Dabora et al. (1965) and Sichel (1966) among others, depends on the ratio of the reaction zone thickness $\Delta$ to the cloud height $h$. While this effect will not be considered in the analysis below, it may be important in the case of detonations in heterogeneous fuel-oxidizer mixtures which often have extensive reaction zones.

Despite these difficulties, the plane configuration provides a useful means of studying fuel-air explosions experimentally under well controlled conditions. Plane detonations have been initiated in $4 \times 4 \times 20 \mathrm{ft}$ rectangular polyethylene bags filled with various fuel-air mixtures. The critical energy required for direct initiation has been determined (Collins and Parsons, 1973), and as described in detail below, the pressure and ground impulse have been determined. For the gases used in these experiments, the critical radius $r^{*} \sim 0(2 \mathrm{ft})$; hence, the detonation induced in the bag propagates mainly as a plane wave with side relief as shown in Fig. 2. This behavior has been verified by photographic observation. Theoretical expressions for the pressure signature and ground impulse

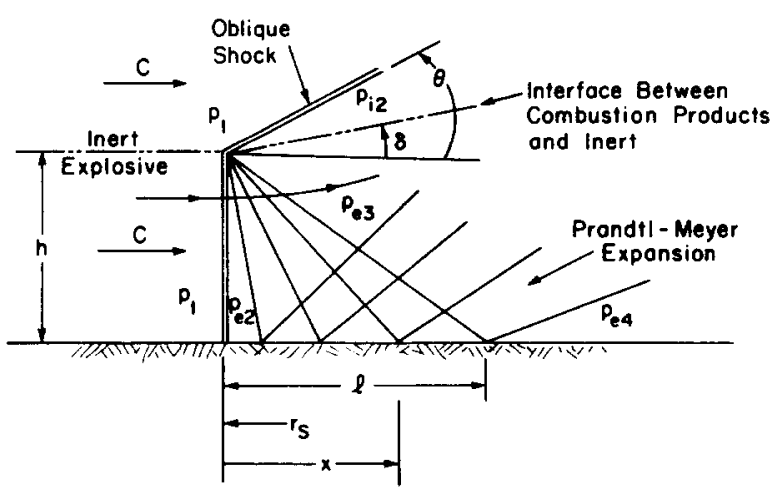

Fig. 2. Interaction between the products of detonation and the inert boundary-coordinates fixed on the detonation. 
developed by such waves will now be derived in order to explain the experimental results.

\section{Analysis}

A plane detonation wave propagating through a fuel-air cloud of width $h$ is considered as shown in detail in Fig. 2 with respect to coordinates fixed to the wave. The pressure behind the detonation drops from $p_{e 2}$ to $p_{e 3}$ across an expansion wave as the combustion products are deflected through the angle $\delta$. The molecular weight of the explosive and inert will be of the same order for a fuel-air mixture bounded by air; hence, the wave induced in the inert is an oblique shock. The strength of the expansion is established by equating the pressure and flow deflection at the interface between the inert gas behind the oblique shock and the combustion products behind the detonation. The expansion wave is reflected from the ground plane where the pressure drops from the CJ value $p_{e 2}$ to $p_{e 4}$ at a distance $l$ from the detonation front. Behind the expansion, where $r<\left(r_{s}-l\right)$, the pressure will be only slightly above atmospheric, and ultimately will be reduced by further reflections from the combustion product-inert interface. The contribution of this region to the ground impulse is, therefore, neglected. The perfect gas equation of state is assumed with suitable values of the ratios of specific heats $\gamma_{e 2}$ and $\gamma_{i 1}$ chosen for the combustion products and the bounding inert gas.

Since the Mach number behind the CJ detonations, $M_{e 2}=1.0$, the deflection angle $\delta$ and the Mach number $M_{e 3}$ downstream of the expansion are related by (Liepmann and Roshko, 1957)

$$
\delta=\left[\frac{\gamma_{e 2}+1}{\gamma_{e 2}-1}\right]^{1 / 2} \tan ^{-1}\left[\frac{\gamma_{e 2}-1}{\gamma_{e 2}+1}\left(M_{e 3}^{2}-1\right)\right]^{1 / 2}-\tan ^{-1}\left[M_{e 3}^{2}-1\right]^{1 / 2}
$$

On the other hand across the oblique shock in the bounding gas

$$
\delta=\tan ^{-1}\left[2 \cot \theta \frac{M_{i 1}^{2} \sin ^{2} \theta-1}{M_{i 1}^{2}\left(\gamma_{i 1}+\cos 2 \theta\right)+2}\right]
$$

and by equating (1) and (2) a single relation between $M_{e 3}$ and the shock angle $\theta$ is obtained. $M_{i 1}$ is the ratio of the detonation velocity $C$ and the speed of sound in the undisturbed inert gas.

An additional relation between the shock angle $\theta$ and $M_{e 3}$ is obtained by equating the pressures on the two sides of the inert-combustion product interface. The pressure ratio across the oblique shock will be

$$
\frac{p_{i 2}}{p_{1}}=1+\frac{2 \gamma_{i 1}}{\gamma_{i 1}+1}\left(M_{i 1}^{2} \sin ^{2} \theta-1\right)
$$

If the gases ahead of and behind the detonation satisfy the perfect gas equation of state, it is readily shown that (Sedov, 1959; Nicholls et al. 1972) 


$$
\frac{p_{e 2}}{p_{1}}=\frac{1+\gamma_{e 1} M_{e 1}^{2}}{\gamma_{e 2}+1}
$$

The simplicity of (4) is deceptive since the parameters $M_{e 1}$ and $\gamma_{e 2}$ can be determined only after the equilibrium composition of the combustion products has been calculated. The computer code of Gordon and McBride (1971) has been used for this purpose here. Downstream of the detonation the combustion products are assumed to be chemically frozen. Then the flow across the expansion wave will be isentropic so that

$$
\frac{p_{e 3}}{p_{e 2}}=\left[\frac{(1 / 2)\left(\gamma_{e 2}+1\right)}{1+(1 / 2)\left(\gamma_{e 2}-1\right) M_{e 3}^{2}}\right]^{\gamma_{e 2} /\left(\gamma_{e 2}-1\right)} .
$$

Now using

$$
\frac{p_{i 2}}{p_{1}}=\left(\frac{p_{e 2}}{p_{1}}\right)\left(\frac{p_{e 3}}{p_{e 2}}\right)
$$

together with (3), (4) and (5) yields the required relation between $M_{e 3}$ and $\theta$.

The simultaneous solution of eqns (1), (2) and (6) was determined iteratively. Once $M_{e 3}$ is known, the deflection angle $\delta$, the interface pressure $p_{e 3}$ and the details of the expansion wave are readily determined. The pressure variation along the ground plane due to the reflection of the expansion wave was determined using the method of characteristics as described, for instance, by Liepmann and Roshko (1957). The reflection of an expansion wave at a solid surface is a standard problem of gas dynamics. Calculations were made for methane air and MAPP-air mixtures for equivalence ratios $\phi$ in the range $0.6 \leq \phi \leq 1.6$ as representative of hydrocarbon fuels, even though the detonability of $\mathrm{CH}_{4}$ air is questionable. Remarkably, over this range the deflection angle $\delta$ and the shock angle $\theta$. were found to remain essentially constant at $\delta=20^{\circ}$, $\theta=29^{\circ}$ for both mixtures. The length $l$ of the reflection zone (Fig. 2) scales with the height $h$ of the fuel oxidizer cloud, and as a consequence of the above results, the ratio $l / h$ has a constant value $l / h=2.90$. A further consequence is that for the mixtures considered, the dimensionless pressure $p / p_{e 2}$ is a universal function of the dimensionless variable

$$
\xi=\frac{x}{h}=\frac{r_{s}-r}{h}
$$

where $x$ is the distance of a given point from the wave front as shown in Fig. 2. The variation of $\left(p / p_{e 2}\right)$ with $\xi$ is shown in Fig. 3 and tabulated in Table 1 . The composition of the MAPP gas used in the calculation is presented in Table 2.

With the pressure distribution behind the detonation known, it now is possible to compute the impulse $I$, per unit area at a fixed distance $r$ from the center of initiation. $r$ is assumed sufficiently large that the steady expansion wave flow pattern described above is fully established. The impulse $I_{s}$ will depend on the relative values of $r$, and the detonation radius $r_{s}$, and on the length 


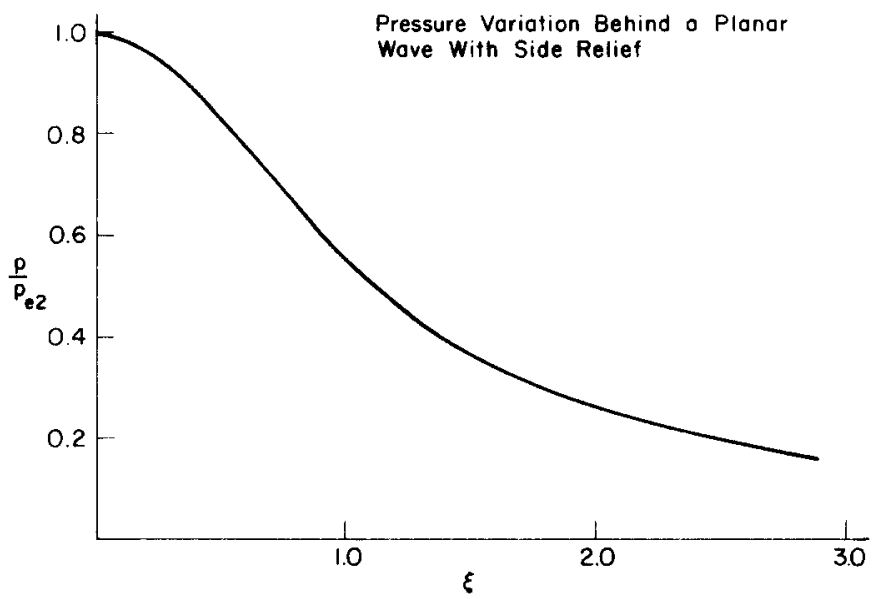

Fig. 3. The variation of $p / p_{e 2}$ with $\xi$.

Table 1. The dimensionless pressure $p / p_{c 2}$ and the universal impulse function $U_{s}(\xi)^{*}$

\begin{tabular}{cllllllll}
\hline$\xi$ & $p / p_{e 2}$ & $U_{s}(\xi)$ & $\xi$ & $p / p_{e 2}$ & $U_{s}(\xi)$ & $\xi$ & $p / p_{e 2}$ & $U_{s}(\xi)$ \\
\hline 0.0 & 1.00 & 0.0 & 1.1 & 0.518 & 0.875 & 2.2 & 0.232 & 1.250 \\
0.1 & 0.995 & 0.100 & 1.2 & 0.462 & 0.924 & 2.3 & 0.220 & 1.272 \\
0.2 & 0.970 & 0.198 & 1.3 & 0.425 & 0.968 & 2.4 & 0.210 & 1.294 \\
0.3 & 0.935 & 0.293 & 1.4 & 0.390 & 1.009 & 2.5 & 0.200 & 1.314 \\
0.4 & 0.890 & 0.384 & 1.5 & 0.362 & 1.046 & 2.6 & 0.190 & 1.334 \\
0.5 & 0.840 & 0.471 & 1.6 & 0.340 & 1.082 & 2.7 & 0.180 & 1.352 \\
0.6 & 0.785 & 0.552 & 1.7 & 0.315 & 1.114 & 2.8 & 0.170 & 1.370 \\
0.7 & 0.730 & 0.628 & 1.8 & 0.295 & 1.145 & 2.9 & 0.160 & 1.386 \\
0.8 & 0.670 & 0.698 & 1.9 & 0.278 & 1.173 & & & \\
0.9 & 0.612 & 0.762 & 2.0 & 0.260 & 1.200 & & & \\
1.0 & 0.560 & 0.821 & 2.1 & 0.247 & 1.226 & & & \\
\hline
\end{tabular}

*Computed numerically from eqn (11)

Table 2. MAPP gas composition used in the impulse calculations

\begin{tabular}{lc}
\hline \multicolumn{1}{c}{ Constituents } & Percentage \\
\hline Methyl acetylene $\left(\mathrm{C}_{3} \mathrm{H}_{4}\right)$ & 35.91 \\
Propadiene $\left(\mathrm{C}_{3} \mathrm{H}_{4}\right)$ & 24.48 \\
Propane $\left(\mathrm{C}_{3} \mathrm{H}_{8}\right)$ & 18.58 \\
C compounds & 13.74 \\
Propylene $\left(\mathrm{C}_{3} \mathrm{H}_{6}\right)$ & 7.29 \\
\hline
\end{tabular}

Molecular weight: $\mathbf{4 3 . 4 7 .}$

Stoichiometric \% MAPP in air by volume: 4.434. 


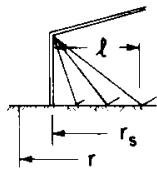

$r_{s}<r$

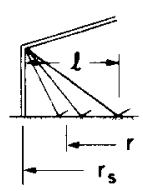

$r_{s}>r>\left(r_{s}-l\right)$

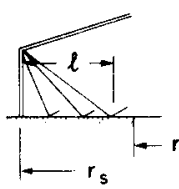

$r<\left(r_{s}-\ell\right)$

Fig. 4. Position of the detonation with respect to a fixed point $r$ on the ground plane.

$l$ of the reflection zone. The three cases which must be considered are shown in Fig. 4. Before the wave reaches position $r, I_{s}=0$. While the wave and the attached expansion wave are passing over the position $r$, i.e. while $r_{s}>r>r_{s}-l$, the impulse per unit area will be

$$
I_{s}=\int_{t_{r}}^{t}\left(p-p_{1}\right) \mathrm{d} t=p_{e 2} \int_{t_{r}}^{t}\left(\frac{p}{p_{e 2}}-\frac{p_{1}}{p_{e 2}}\right) \mathrm{d} t
$$

Here the arrival time $t_{r}=(r / C)$ is the time when $r_{s}=r$. When $r<r_{s}-l$, the impulse $I_{s}$ remains constant at

$$
I_{s}=p_{e 2} \int_{t_{r}}^{t_{r}+(U C)}\left(\frac{p}{p_{e 2}}-\frac{p_{1}}{p_{e 2}}\right) \mathrm{d} t
$$

since, as already indicated, the effect of the region behind the reflected expansion is neglected.

Now using the definition of $\xi$ in (7) and the relation $\mathrm{d} r_{s}=C \mathrm{~d} t$, the integrals in (8) can be expressed in terms of $\xi$ so that

$$
I_{s}=p_{e 2}\left(\frac{h}{C}\right) \int_{0}^{\xi}\left[\frac{p}{p_{e 2}}-\frac{p_{1}}{p_{e 2}}\right] \mathrm{d} \xi=p_{1}\left(\frac{h}{C}\right) \frac{p_{e 2}}{p_{1}}\left[U_{s}(\xi)-\frac{p_{1}}{p_{e 2}} \xi\right]
$$

with $U_{s}(\xi)$ the universal function

$$
U_{s}(\xi)=\int_{0}^{\xi} \frac{p}{p_{e 2}}(\xi) \mathrm{d} \xi
$$

which is plotted in Fig. 5 and tabulated in Table $1 . U_{s}(\xi)$ can also be represented quite accurately by the expression

$$
U_{s}(\xi)=1.525[1-\exp (-0.773 \xi)]
$$

as shown in Fig. 5. At the end of the reflection region $\xi=l / h=2.90$ and $U_{s}(l / h)=1.386$; hence when $r<r_{s}-l$, the impulse $I_{s}$ will have the constant value

$$
I_{s}=p_{1}\left(\frac{h}{C}\right) \frac{p_{e 2}}{p_{1}}\left[1.386-2.90\left(\frac{p_{1}}{p_{e 2}}\right)\right]
$$




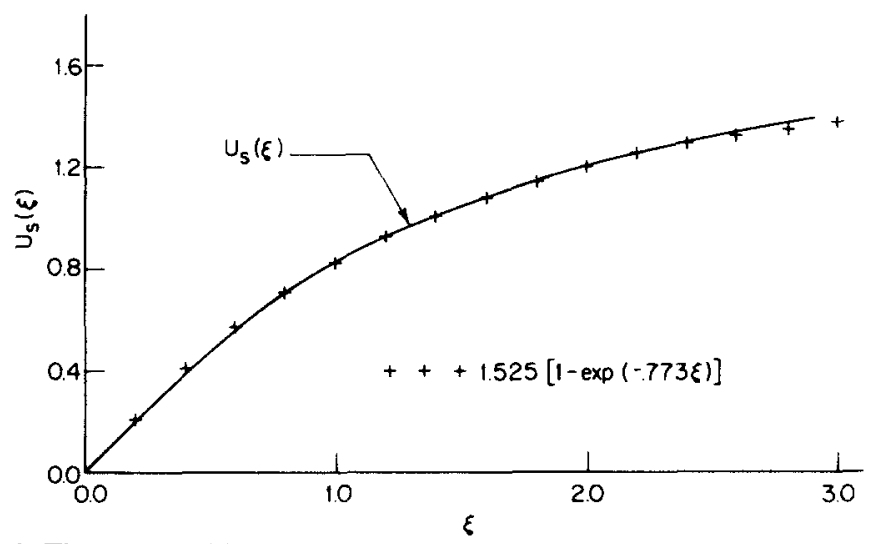

Fig. 5. The universal impulse function $U_{s}(\xi)$ for a plane wave with side relief.

Equations (10) and (13) provide very simple expressions for the impulse per unit area at some distance $r$ from blast center. While the results have specifically been calculated for MAPP-air and $\mathrm{CH}_{4}$-air mixtures, it does not appear unreasonable that (10) and (13) can be used for most hydrocarbon-air detonations in the range $0.6 \leq \phi \leq 1.6$. Once the expansion wave behind the detonation is fully developed, the flow becomes independent of the distance $r$ from blast center, which explains why $I_{s}$ is also independent of $r$. It is of interest that $I_{s}$ is directly proportional to the ratio $h / C$, the time it would take for the detonation to propagate across the fuel cloud.

For very large values of $(h / r)$ the detonation will behave like one in a duct with rigid boundaries. Then, as mentioned above, the detonation will be followed by a Taylor expansion instead of a centered expansion wave propagating inward from the inert boundary. In this case the ground impulse generated per unit area at a distance $r$ from blast center has been computed by Nicholls et al. (1972). With a Taylor expansion behind the wave the impulse is given by the expression

$$
I_{T}=p_{1}(r / C)\left[\frac{p_{e 2}}{p_{1}} U_{T}(\tau)-(\tau-1)\right]
$$

for $t>(r / C)$. Here $\tau=t / t_{r}=t /(r / C)$, and $I_{T}=0$ when $t<t_{r} U_{T}(\tau)$ is a universal impulse function which is given in Table 4 both as a function of $\tau$ and of the variable $\lambda=r / r_{s}=\tau^{-1}$.

Now the impulse $I_{T}$ is no longer independent of the distance $r$ from blast center. Because of the pressure plateau behind the Taylor Wave the impulse continues to increase with time instead of reaching a constant value. Thus when $\tau \gg 1$ it is shown by Nicholls et al. (1972) that

$$
I_{T} \cong p_{1}(r / C)\left[0.358\left(p_{e 2} / p_{1}\right)-1\right] \tau=p_{1}\left[0.358\left(p_{e 2} / p_{1}\right)-1\right] t .
$$

In this limiting case $I_{T}$, thus, varies directly with time, and no longer depends on the radius $r$.

It is possible to estimate the minimum value of $h / r_{c}$ for a fuel cloud of radius 
$r_{c}$ below which the effect of side relief must be considered by assuming that a $\mathrm{C}-\mathrm{J}$ detonation is initiated almost instantly at blast center. Then a signal from the inert boundary will propagate to the ground surface in approximately the time $\left(h / a_{2}\right)$ required for a sound wave to travel across the cloud at the speed of sound $a_{2}$ behind the detonation. During this period the detonation will travel a distance $r=C t=C\left(h / a_{2}\right)$ so that $(h / r) \cong a_{2} / C$, when the effect of side relief first reaches the ground plane. For a $C-J$ detonation it is readily shown that $a_{2} / C=\rho_{1} / \rho_{2}=$ $\gamma_{e 2} /\left(\gamma_{e 2}+1\right)$. For most hydrocarbon detonations $\gamma_{e 2} \sim 1.18$ so that, finally, $(h / r) \cong$ $0.54 \sim 1 / 2$. For clouds with $\left(h / r_{c}\right) \geq 1 / 2$ the detonation reaches the cloud boundary before side relief becomes important, and the impulse will then depend on reflections from the cloud boundary. When $\left(h / r_{c}\right) \leqslant 1 / 2$ side relief must be taken into account.

\section{Experimental measurements}

Experiments were conducted using $4 \times 4 \times 20 \mathrm{ft}$ supported polyethylene bags. Complete fuel-air mixing was obtained with a fan. Disc shaped $85 \mathrm{gm}$ PETN based Dupont "detasheet" explosive initiators were placed at one end of the bag. Kisler Corporation Model 202C series piezoelectric pressure transducers mounted level with the ground along the center of the bags were used to measure the variation of the pressure with time. The transducers were placed at distances of 12,13 and $14 \mathrm{ft}$ from the initiator. Signals were conditioned and then recorded by a Honeywell Corporation Model 7620 wide band magnetic tape recorder. The impulse, obtained by integrating the pressure signal, was also recorded. The average temperature within the enclosure for all tests was $36 \pm 3^{\circ} \mathrm{C}$. For each test the shock wave arrival time, maximum detonation pressure, and the total impulse were recorded.

Tests were made using stoichiometric mixtures of air and MAPP gas (Table 2), 1.3 butadiene $\left(\mathrm{C}_{4} \mathrm{H}_{6}\right)$ and propylene oxide $\left(\mathrm{C}_{3} \mathrm{H}_{6} \mathrm{O}\right)$. Typical pressure and impulse traces, recorded for the MAPP-air mixture, are reproduced in Figs. 6(a) and $6(\mathrm{~b})$. The second small pressure peak, appearing in all the traces, is due to the arrival of the reflection of the detonation from a metal plate at the end of the bag, and also results in a slight jump in the final value of the impulse. This effect has been neglected in the analysis of the data. In interpreting the data it is important to recognize that expansion waves propagate into the combustion products not only from the top surface of the bag, $4 \mathrm{ft}$ from the pressure transducers, but also from the sides at a distance of $2 \mathrm{ft}$ from the transducers. Consequently, the data has been treated as equivalent to that from a cloud of height $h=2.0 \mathrm{ft}$. The interaction of the waves from the top and sides of the bag will result in a complex three dimensional flow near the center. Comparison of the data with the analysis developed above indicates that this interaction, which has been neglected here, has a small effect on the pressure and impulse recorded on the bag floor.

The results of all the experiments are summarized in Table 3. Both the measured and calculated values of the pressure $p_{e 2}$ and the impulse $I_{s}$ are presented. The measured values of $p_{e 2}$, which were taken as the peak values from the pressure traces, tend to be consistently above the theoretical values by 
Table 3. Experimental and theoretical values of total impulse and peak pressure

\begin{tabular}{|c|c|c|c|c|c|}
\hline \multicolumn{6}{|c|}{ 1,3 Butadiene: $p_{e 2} / p_{1}=18.68 ; C=5.938 \mathrm{ft} / \mathrm{sec}$} \\
\hline & & $\begin{array}{c}p_{e 2} \\
\text { psia }\end{array}$ & $\begin{array}{c}p_{e 2} \\
\text { psia }\end{array}$ & $\begin{array}{l}\left(I_{s}\right)_{\max } \\
\text { meas. }\end{array}$ & $\begin{array}{l}\left(I_{s}\right)_{\max } \\
\text { theory }\end{array}$ \\
\hline Shot & Station & meas. & theory & psi-msec & psia-msec \\
\hline 2 & 2 & 328.4 & 274.7 & 110.0 & 113.9 \\
\hline 2 & 3 & 331.3 & $\downarrow$ & 100.2 & $\downarrow$ \\
\hline \multicolumn{6}{|c|}{ MAPP: $p_{e 2} / p_{1}=18.62 ; C=5953 \mathrm{ft} / \mathrm{sec}$} \\
\hline & & $\begin{array}{c}p_{e 2} \\
\text { psia }\end{array}$ & $\begin{array}{l}p_{e 2} \\
\text { psia }\end{array}$ & $\begin{array}{l}\left(I_{s}\right)_{\max } \\
\text { meas. }\end{array}$ & $\begin{array}{l}\left(I_{s}\right)_{\max } \\
\text { theory }\end{array}$ \\
\hline Shot & Station & meas. & theory & psi-msec & psia-msec \\
\hline 1 & 1 & 321.2 & 273.7 & 109.5 & 113.1 \\
\hline 2 & 1 & 279.4 & & 99.8 & \\
\hline 2 & 2 & 363.3 & & 95.8 & \\
\hline 3 & 2 & 322.7 & & 90.1 & \\
\hline 3 & 3 & 273.7 & & 102.7 & $\downarrow$ \\
\hline \multicolumn{6}{|c|}{ Propylene oxide: $p_{e 2} / p_{1}=19.881 ; C=6053 \mathrm{ft} / \mathrm{sec}$} \\
\hline & & $\begin{array}{c}p_{e 2} \\
\text { psia }\end{array}$ & $\begin{array}{c}p_{e 2} \\
\text { psia }\end{array}$ & $\begin{array}{l}\left(I_{s}\right)_{\max } \\
\text { meas. }\end{array}$ & $\begin{array}{l}\left(I_{s}\right)_{\max } \\
\text { theory }\end{array}$ \\
\hline Shot & Station & meas. & theory & psi-msec & psia-msec \\
\hline 1 & 3 & 284.5 & 292.2 & 116.5 & 119.8 \\
\hline 2 & 1 & 343.7 & & 113.0 & \\
\hline 2 & 3 & 325.7 & $\downarrow$ & 110.1 & $\downarrow$ \\
\hline
\end{tabular}

as much as $33 \%$. This divergence has not been explained but could be caused by ringing of the transducers or by interaction between the bottom of the bag and the transducers on the ground. The impulse provides a more significant basis for comparing theory and experiment, since it represents the integrated effect of the pressure, and here the agreement appears to be quite good.

The pressure trace in Fig. 6(a) has been reproduced in Fig. 7 along with the theoretically computed pressure. For the theoretical curve the variable $\xi$ was converted to time using $h=2.0 \mathrm{ft}$ and the theoretical detonation velocity $C=$ $5953 \mathrm{ft} / \mathrm{sec}$ for stoichiometric MAPP air. Except for the initial part of the trace where measured pressure drops sharply and appears to oscillate, the agreement

Table 4. The universal impulse function $U_{T}(\tau)$

\begin{tabular}{cccccc}
\hline$\lambda$ & $\tau$ & $U(\tau)$ & $\lambda$ & $\tau$ & $U(\tau)$ \\
\hline 1.00 & 1.000 & 0.000 & 0.60 & 1.667 & 0.426 \\
0.95 & 1.050 & 0.050 & 0.50 & 2.000 & 0.556 \\
0.90 & 1.110 & 0.100 & 0.40 & 2.500 & 0.740 \\
0.85 & 1.178 & 0.152 & 0.30 & 3.333 & 1.046 \\
0.80 & 1.250 & 0.203 & 0.20 & 5.000 & 1.659 \\
0.75 & 1.330 & 0.256 & 0.10 & 10.000 & 3.500 \\
0.70 & 1.429 & 0.310 & & & \\
\hline
\end{tabular}


between theory and experiment is excellent. The corresponding measured and theoretical impulse variation is shown in Fig. 8. Here, too, agreement is extremely good. While all the pressure traces and the corresponding impulse showed similar behavior, detailed comparison was restricted to the case shown in Figs. 7 and 8 , since the most significant parameter is the total impulse $I_{s}$ presented in Table 3.

The impulse $I_{T}$ computed using eqn (14) for $r=14 \mathrm{ft}$ is also shown in Fig. 8. It is clear that in this case the effect of side relief is dominant, and that the impulse based on the Taylor expansion is much too high after about $0.4 \mathrm{msec}$.

The pressure signature should be independent of detonation radius $r_{s}$ for a fully established fuel-air explosion with side relief. This behavior appears to be verified by Fig. 9 which shows the pressure traces in MAPP-air 13 and $14 \mathrm{ft}$ from the initiator with the onset of the pressure rise, in each case, placed at the origin to facilitate comparison. The reflected wave pressure rise occurs earlier in the upper trace for station 3 , which is one foot closer to the steel end plate.

\section{Discussion of results}

A simple theory for computing the pressure signature and impulse within the cloud of a plane fuel-air explosion has been developed. The key element of the theory consists of no more than computing the reflection of a centered expansion wave at a solid wall. The theoretical results were in good agreement with

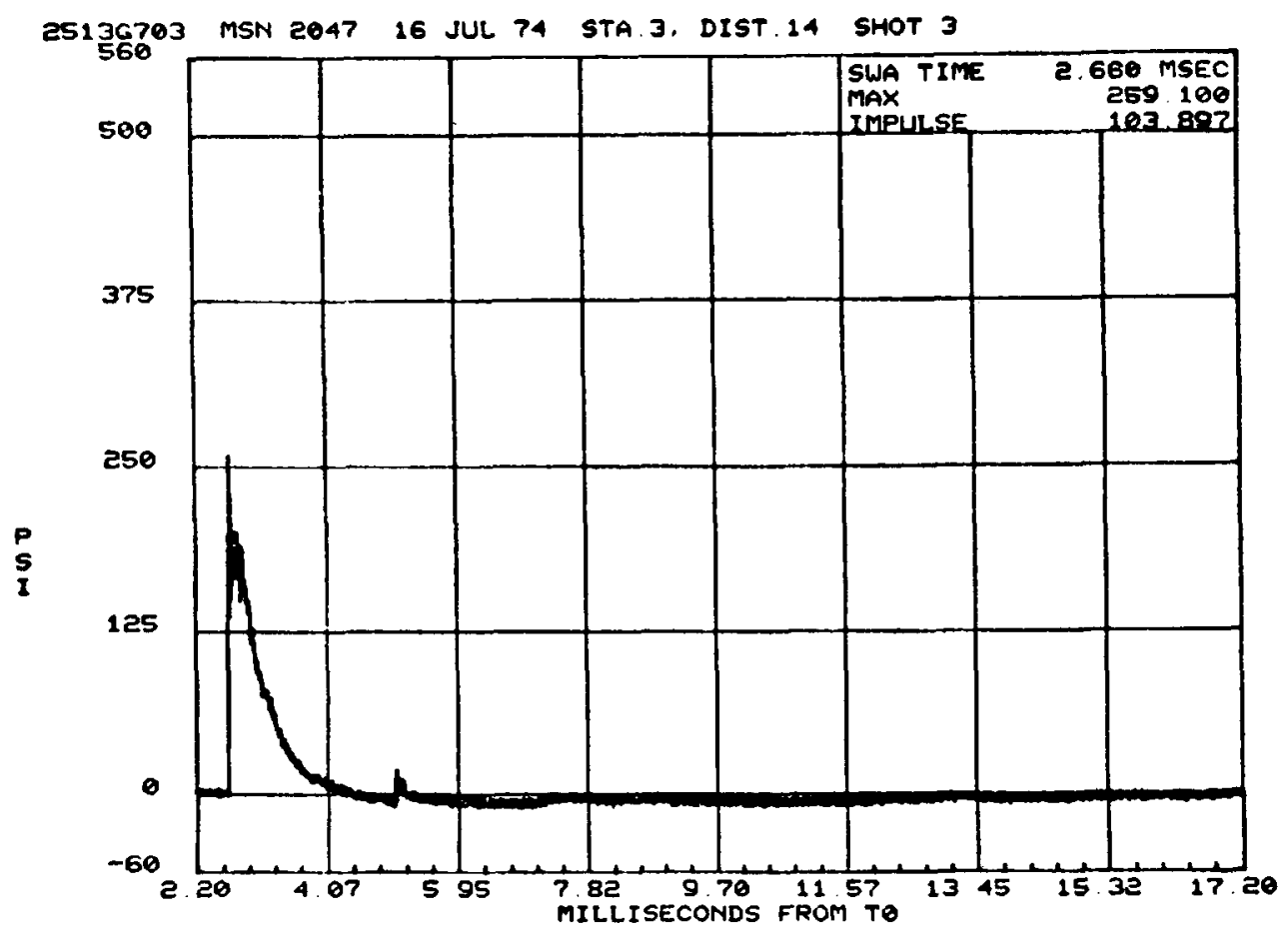

Fig. 6(a). Experimental pressure trace for stoichiometric MAPP-air; wave moving from right to left. 


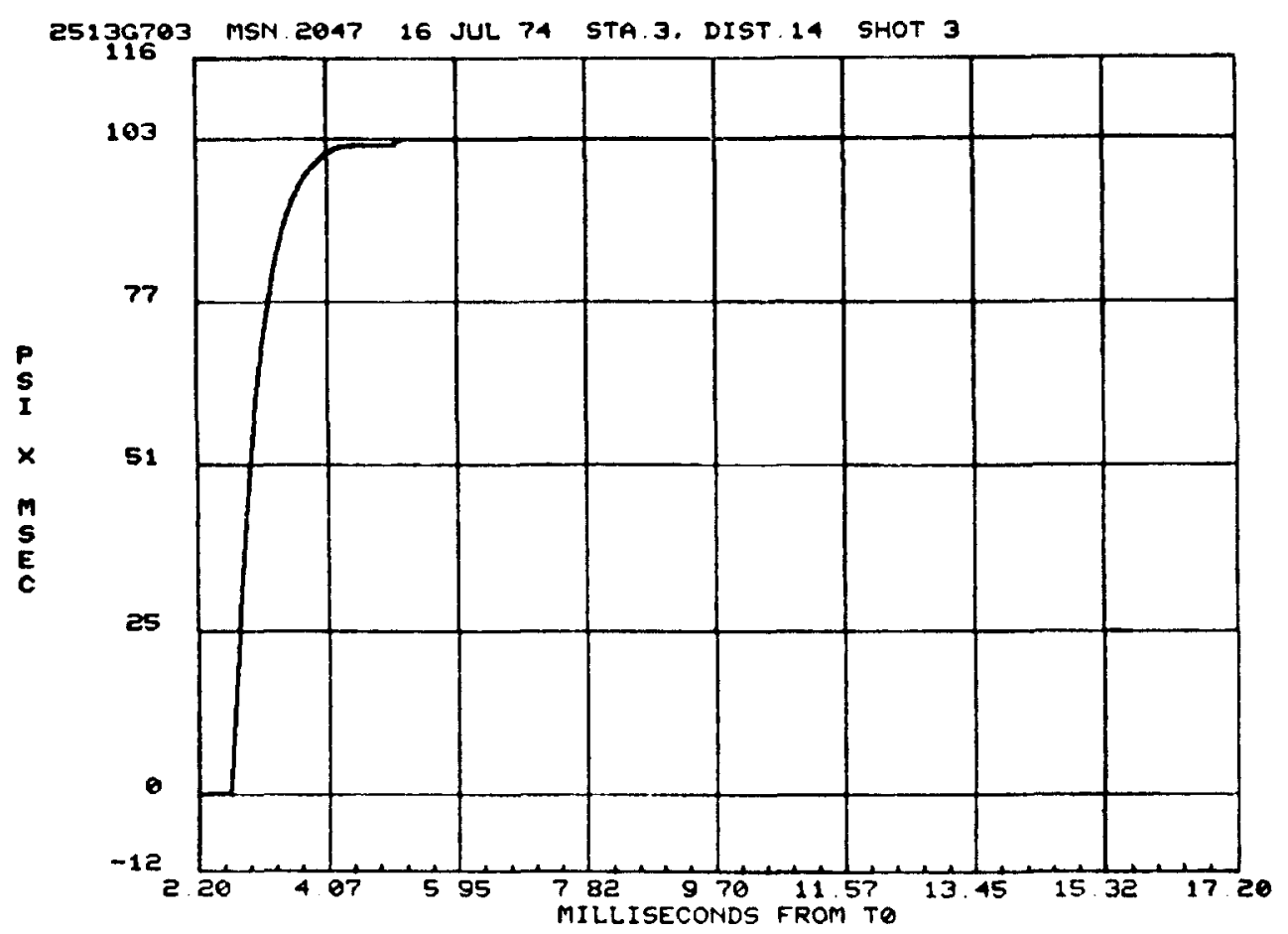

Fig. 6(b). Experimental impulse trace for stoichiometric MAPP-air; wave moving from right to left.

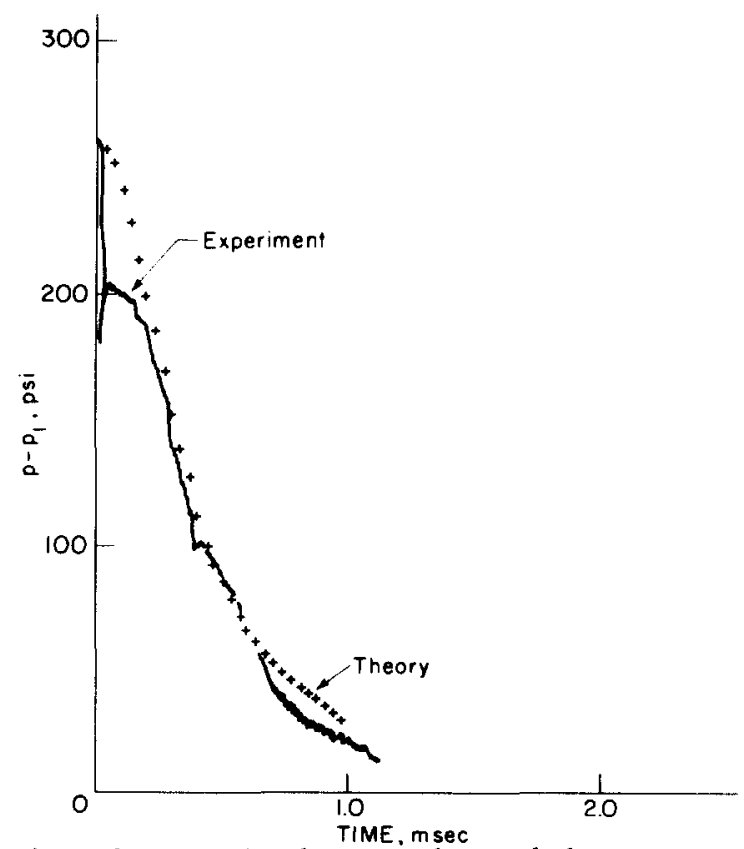

Fig. 7. Comparison of measured and computed ground plane pressure for MAPP-air [Data of Fig. 6(a)]. 


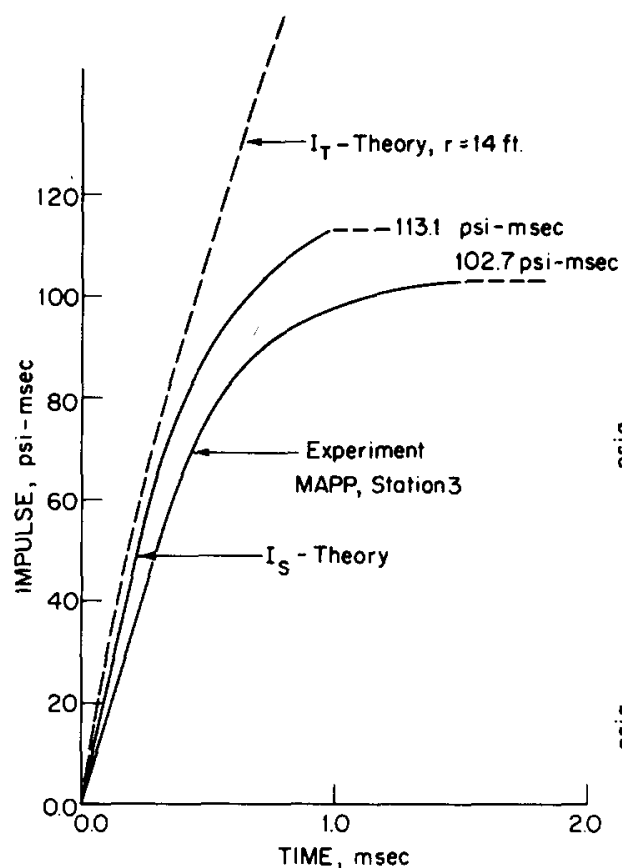

Fig. 8.
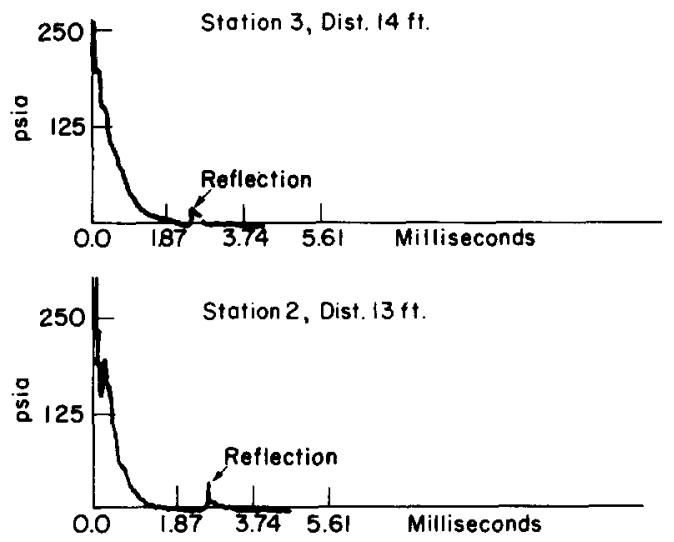

Fig. 9.

Fig. 8. Comparison of measured and computed ground impulse per unit area for MAPP-air [Data of Fig. 6(b)].

Fig. 9. Experimental pressure traces plotted using a common origin for the time scale.

measured pressure signatures and ground impulse. Since only one value of $h$ was used in the experiments it is of course possible that this agreement was fortuitous; however the results are certainly encouraging. Interestingly, the normalized pressure and impulse computed for methane air and MAPP-air were almost independent of the mixture composition over a wide range of equivalence ratios. It appears likely that this result remains valid for hydrocarbon-air detonations with fuels other than methane and MAPP gas.

The comparison of the theory described above with a carefully controlled experiment is the main contribution of the present paper. The results suggest that many aspects of fuel-air explosions can be studied using relatively simple analytical methods without immediate recourse to numerical computation.

Acknowledgements-Part of this work was supported by the Air Force Armament Laboratory, Eglin Air Force Base, Florida, under Contract F08635-75-C-0129. Mr. Cheng Hu carried out many of the calculations while a graduate student at The University of Michigan.

\section{References}

Bach G. G., Knystautas R. and Lee J. H. (1971) Initiation criteria for diverging gaseous detonations. 13th Symp. (Int.) on Combustion, The Combustion Institute, Pittsburgh, Pennsylvania, 1097.

Collins P. M. and Parsons G. H. (1973) Detonation initiation in unconfined fuel-air mixtures. Proc. of the Conf. on Mechanisms of Explosion and Blast Waves, Joint Technical Coordinating Group 
for Air Launched Non-Nuclear Ordnance Working Party for Explosives, Naval Weapons Station, Yorktown, Virginia, 13-15 November.

Dabora E. K., Nicholls J. A. and Morrison R. B. (1965) The influence of the compressible boundary on the propagation of gaseous detonations, 10th Symp. (Int.) on Combustion, p. 817. The Combustion Institute, Pittsburgh, Pennsylvania.

Fishburn Barry D. (1976) Some aspects of blast from fuel-air explosives. Acta Astronautica 3 , 1049-1065.

Gordon S. and McBride B. (1971) Computer program for calculation of complex chemical equilibrium compositions, rocket performance, incident and reflected shocks, and Chapman Jouguet detonations. National Aeronautics and Space Administration SP 273.

Lee J. H. (1972) Gasdynamics of detonations. Astronautica Acta 17, 455-466.

Liepmann H. W. and Roshko A. (1957) Elements of Gasdynamics. Wiley, New York.

Nicholls J. A., Fry R. S., Glass D. R., Sichel M., Vander Schaaf J. and Sternstein A. J. (1972) Fundamental aspects of unconfined explosions. Tech. Rep. AFATL-TR-72-49, US Air Force Armament Lab., Eglin AFB, Florida, U.S.A.

Sichel M. (1966) A hydrodynamic theory for the propagation of gaseous detonations through charges of finite width. AIAA J. 4, 264.

Sichel M. and Hu Cheng (1973) The impulse generated by blast waves propagating through combustible mixtures. Proc. of the Conf. on Mechanisms of Explosion and Blast Waves, Joint Technical Coordinating Group for Air Launched Non-Nuclear Ordnance Working Party for Explosives, Naval Weapons Station, Yorktown, Virginia, 13-15 November.

Sedov L. I. (1959) Similarity and Dimensional Methods in Mechanics, 4th Edn. Academic Press, New York.

Taylor G. I. (1958) Gas dynamics of combustion and detonation, Sec. G in Fundamentals of Gas Dynamics, Vol. III, Princeton Ser. in High Speed Aerodynamics and Jet Propulsion (Ed. by H. Emmons). Princeton University Press, Princeton, N.J.

Zeldovitch Ya. (1942) Distribution of pressure and velocity in the products of a detonating explosion and in particular in the case of a spherical propagation of the detonation waves. J. Exp. Theor. Phys., (USSR) 12, 389-406. 